\title{
Yeast GCN4 as a probe for oncogenesis by AP-1 transcription factors: transcriptional activation through AP-1 sites is not sufficient for cellular transformation
}

\author{
Salvatore Oliviero, ${ }^{1,3}$ Gregory S. Robinson, ${ }^{1,2}$ Kevin Struhl, ${ }^{1}$ and Bruce M. Spiegelman ${ }^{1,2}$ \\ ${ }^{1}$ Department of Biological Chemistry and Molecular Pharmacology, Harvard Medical School, Boston, Massachusetts 02115 \\ USA; ${ }^{2}$ Division of Cellular and Molecular Biology, Dana-Farber Cancer Institute, Boston, Massachusetts 02115 USA; \\ ${ }^{3}$ Dipartimento di Biologia, Universita degli Studi di Padova, via Trieste, 75-35121 Padova, Italy
}

The Jun and Fos oncoproteins belong to the AP-1 family of transcriptional activators and are believed to induce cellular transformation by inappropriately activating genes involved in cell replication. To determine whether transcriptional activation through AP-1 sites is sufficient for transforming activity, we examined the properties of an autonomous and heterologous AP-1 protein, yeast GCN4, in rat embryo fibroblasts. GCN4 induces transcriptional activation through AP-1 sites but, unlike Jun and Fos, fails to induce cellular transformation, in cooperation with Ha-ras. Jun-GCN4 and Fos-GCN4 homodimers independently induce cellular transformation indicating that the amino-terminal regions of Jun and Fos each contain regulatory functions that are required for oncogenesis but are distinct from generic transcriptional activation domains. In addition, these observations have implications for the nature of the oncogenically relevant target genes that respond to Jun and Fos.

[Key Words: Yeast GCN4; Jun; Fos; AP-1 transcription factors; oncogenesis; cellular transformation]

Received May 21, 1992; revised version accepted July 7, 1992.

$\mathrm{v}$-Fos and v-Jun are oncogenes carried by murine osteosarcoma and avian fibrosarcoma retroviruses, respectively (Curran et al. 1982; Miller et al. 1984; Maki et al. 1987). The cellular homologs of these oncogenes, c-Jun and c-Fos, belong to the AP-1 family of transcription factors that bind specific DNA sequences/consensus TGACTCA), termed AP-1 sites (Bohmann et al. 1987; Distel et al. 1987; Struhl 1987; Angel et al. 1988a; Curran and Franza 1988; Ransone and Verma 1990; Boyle et al. 1991). AP-1 sites are found in many eukaryotic genes and are often associated with transcriptional stimulation in response to a variety of extracellular stimuli, including tumor promoters such as phorbol esters (Angel et al. 1987; Lee et al. 1987; Brenner et al. 1989; Stein et al. 1989; Jonat et al. 1990; Schule et al. 1990; Yang-Yen et al. 1990). AP-1 proteins contain a bZIP DNA-binding domain (Landschulz et al. 1988) consisting of a leucine zipper that mediates dimerization (Kouzarides and Ziff 1988; Neuberg et al. 1989a; Sellers and Struhl 1989) and symmetrically positions the adjacent basic regions for specific interactions with DNA /Agre et al. 1989; Pu and Struhl 1991a,b). AP-1 proteins can form homodimeric and/or heterodimeric complexes with DNA, with individual members of the family having distinct dimeriza- tion specificity. Jun forms weak DNA-binding homodimers and Fos cannot dimerize, whereas Fos-Jun heterodimers bind AP-1 sites with high affinity.

It is generally believed that Fos and Jun contribute to the oncogenic state by binding to AP-I sites and inappropriately activating genes involved in the control of cell proliferation. In this regard, several mutational analyses indicate a strong correlation between AP-1 site-dependent transcriptional activation, cellular transformation (often in cooperation with the activated Ha-ras), and tumor formation (Bohmann and Tjian 1989, Neuberg et al. 1989b; Schuermann et al. 1989; Baichwal and Tjian 1990; Alani et al. 1991; Binetruy et al. 1991; Lucibello et al. 1991; Wisdom et al. 1992). However, Jun and Fos are complex transcriptional regulators that activate or repress transcription, depending on the promoter and cellular factors with which they interact. Such complexities include the existence of multiple Jun-like and Foslike proteins with different functional properties /Chiu et al. 1989; Li et al. 1990; Castellazzi et al. 1991; Doucas et al. 1991; Mumberg et al. 1991; Nakabeppu and Nathans 1991; Wisdom et al. 1992), the ability of the Jun and Fos proteins to regulate their own expression (Angel et al. 1988b; Konig et al. 1989), and the potential of Jun 
and Fos to interact with other proteins involved in transcriptional regulation (Diamond et al. 1990; Jonat et al. 1990; Schule et al. 1990; Yang-Yen et al. 1990; Bengal et al. 1992; Li et al. 1992). It is also unclear whether Fos and/or Jun encode oncogenic-specific functions or whether they cause cellular transformation more indirectly by associating with proteins containing the leucine zipper motif such as other members of the AP-1 family or activating transcription factor-cAMP response element binding (ATF-CREB) proteins. Finally, the physiologically relevant target genes regulated by the Jun and Fos oncoproteins have not yet been identified. Thus, the functional complexities of mammalian AP-1 factors that allow for the exquisite regulation of gene expression make it difficult to determine the mechanisms by which Jun and Fos cause malignant transformation.

In this paper we investigate the role of AP-1 transcriptional activation in the process of oncogenic transformation using a novel approach involving the yeast transcription factor GCN4. GCN4, Jun, and Fos bind to identical DNA sequences (Hill et al. 1986; Bohmann et al. 1987; Distel et al. 1987; Struhl 1987; Angel et al. 1988a; Halazonetis et al. 1988; Nakabeppu et al. 1988), and Jun homodimers or Jun-Fos heterodimers can activate transcription in yeast cells and functionally substitute for GCN4 (Struhl 1988; Oliviero and Struhl 1991). However, GCN4 cannot interact with Jun or Fos (Kouzarides and Ziff 1989; Sellers and Struhl 1989l, and it activates transcription primarily, and probably exclusively, by binding DNA as a homodimer (Pu and Struhl 1991b). Given the long evolutionary distance between yeast and mammals, it seems unlikely that GCN4 will be able to carry out Jun- or Fos-specific regulatory interactions beyond DNAbinding and transcriptional activation. Thus, GCN4 can be viewed as an autonomous and heterologous AP-1 transcription factor whose function should not be subject to the vast regulatory complexities of the Jun-Fos family. By analyzing the oncogenic properties of GCN4 derivatives, as well as GCN4-Jun and GCN4-Fos chimeric proteins, we demonstrate that transcriptional activation through AP-1 sites is not sufficient for cellular transformation. Moreover, we show that the amino-terminal regions of Fos and Jun each encode oncogenic functions that are distinct from generic transcriptional activation domains.

\section{Results}

GCN4 homodimers activate transcription from AP-1 sites in mammalian cells

To investigate whether yeast GCN4 can activate AP-1dependent transcription and induce neoplastic transformation in mammalian cells, the coding region of GCN4 and several derivatives were cloned into the expression vector pMTpn (Fig. 1). To achieve high expression of the protein in mammalian cells, the GCN4 translational initiation region was replaced by an optimal mammalian translational initiation sequence (Kozak 1987). The GCN4 3'-untranslated region was substituted with the

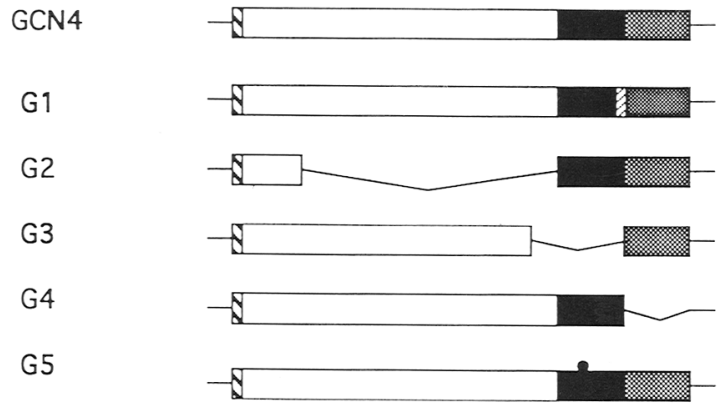

Figure 1. Schematic diagram of GCN4 and GCN4 mutants. GCN4 wild type (GCN4) was modified and/or the specific regions were deleted to generate the mutants G1-G5 (Material and methods). ( ( ) Activation domain; ( $\mathbf{\text { a }}$ ) basic domain; (imerization domain; $(\mathbb{C})$-amino-acid insertion; $(\boldsymbol{D})$ optimal translation start.

untranslated region of the human growth hormone, which increases message stability. Transcriptional activities of the various GCN4 derivatives were assessed in rat embryo fibroblasts (REFs) by transient cotransfection with chloramphenicol acetyltransferase $(C A T)$-reporter constructs (Fig. 2).

GCN4 stimulates transcription from a promoter containing five copies of the AP-1 site from the collagenase gene upstream of the TATA element of the thymidine kinase gene $(5 \times \mathrm{TRE} / \mathrm{tk}-\mathrm{CAT})$. The level of transcription is comparable to that achieved by Jun and/or Fos. Deletion mutants that remove the activation $\{\mathrm{G} 2\}$, DNA-binding $(\mathrm{G} 3)$, or dimerization $(\mathrm{G} 4)$ domains prevent AP-1 site-dependent transcriptional activation as does the N235E substitution in the GCN4 basic region (G5) that severely reduces DNA-binding activity (Pu and Struhl 1991a). Similarly, GCN4 activates transcription of the natural aP2 promoter, which contains one AP-1 binding site, as well as a CCAAT/enhancer-binding protein (C/EBP)-binding site (Herrera et al. 1989). As expected, transcriptional activation by GCN4 requires functionally intact bZIP and activation domains, and it is inhibited by a point mutation in the AP-1 recognition sequence. Thus, GCN4 activates AP-1 site-dependent transcription efficiently in mammalian cells with the same functional domains required for activity in yeast cells.

GCN4 functions primarily (and probably exclusively) as a homodimer in yeast cells (Pu and Struhl 1991b), and it does not form heterodimers with Jun or Fos (Kouzarides and Ziff 1989; Sellers and Struhl 1989). To determine whether GCN4 activity in mammalian cells is the result of homodimer formation, we examined the Gl derivative, which contains a 7 amino acid insertion between the leucine zipper and the basic region (Fig. 1). Gl efficiently binds AP-1 sites as a homodimer but it is unable to form DNA-binding heterodimers with wildtype GCN4; presumably, any heterodimeric interactions with GCN4 or G1 would involve a different (and probably mutually exclusive) set of bZIP proteins (Pu and Struhl 1991 b). The observation that Gl activates tran- 


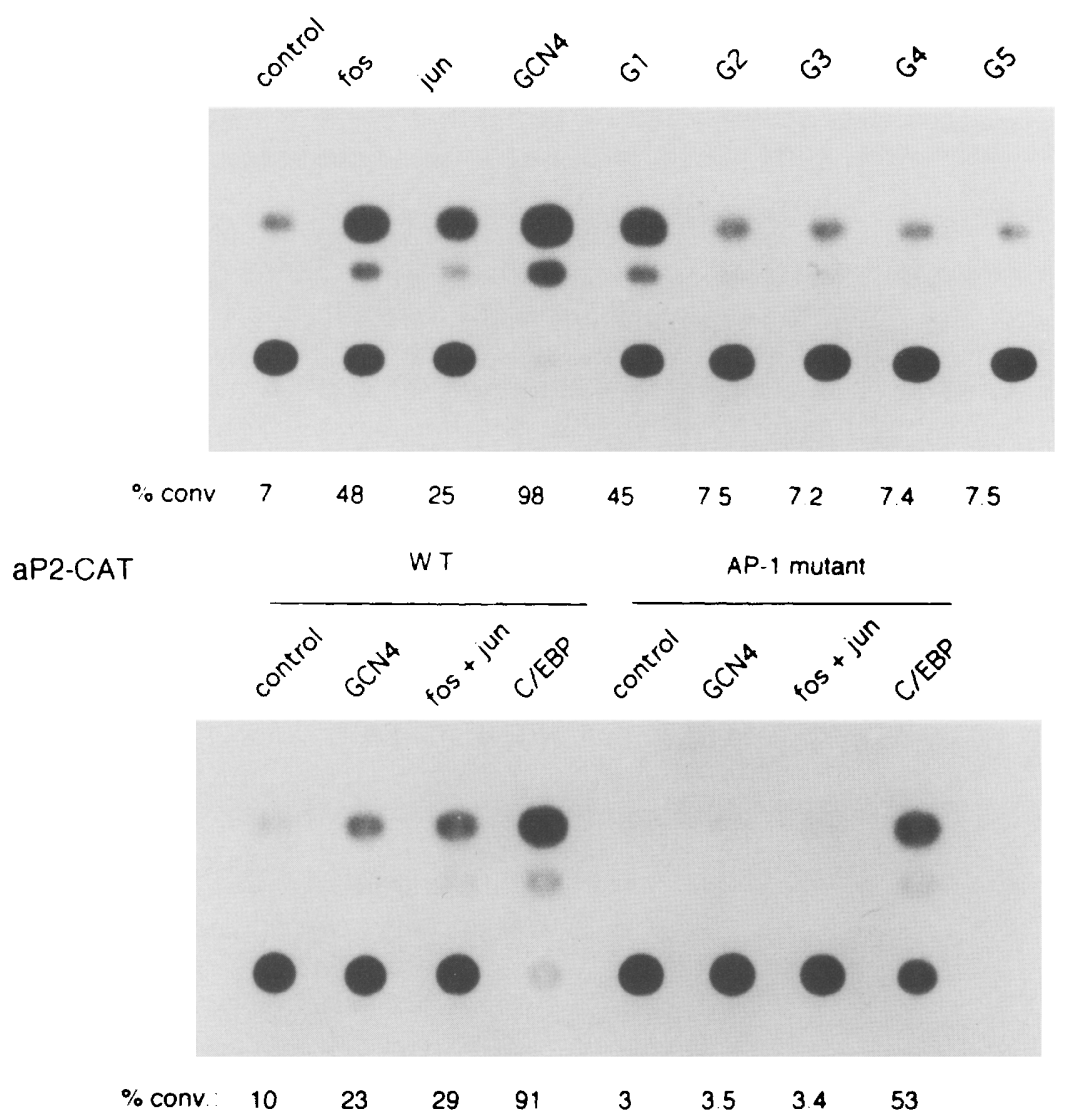

Figure 2. AP-1-dependent transcription occurs in natural or hybrid promoter CAT constructs. (Top) REFs were transiently transfected with the $5 \times \mathrm{TRE} / \mathrm{tk}-\mathrm{CAT}$ construct and $10 \mu \mathrm{g}$ of each plasmid construct, respectively. Cellular extracts were analyzed for CAT activity as described in Material and methods. Three or more independent experiments were completed, and a representitive chromatograph is shown. When the CAT assay is carried out for less time ito ensure linearity), the GI derivative shows $50-70 \%$ of the activity of GCN4. (Bottom) REFs were transiently transfected with Ap2 (WT)-CAT, a construct containing one AP-1 site, or with a construct where the AP-1 consensus sequence has been mutated (mutant). The cellular extracts were assayed, and the transcriptional activity for each is listed. scription to levels comparable to those achieved by GCN4 (Fig. 2) indicates that transcriptional activity is mediated primarily (and probably solely) by homodimers.

Finally, we tested whether GCN4 could activate transcription from AP-1 sites when stably expressed in REFs. We generated several G418-resistant pools of cell lines expressing GCN4, Jun, or Fos and measured transcriptional activity upon transfection with the $5 \times \mathrm{TRE} / \mathrm{tk}-$ CAT plasmid \{Table 1). Cell lines containing GCN4, Jun, and Fos DNAs displayed comparable levels of AP-1 sitedependent activity, whereas control cell lines containing the expression vector showed background levels. As expected, we were unable to recover clones from cells transfected with a vector expressing C/EBP, a transcription factor that inhibits cell replication /Umek et al. 1991). The fact that GCN4, Jun, and Fos can activate transcription in stable cell lines indicates that expression of these proteins is not cytostatic or cytotoxic.

\section{AP-1 transcriptional activation is not sufficient to induce transformation}

We then examined whether GCN4, like Fos and Jun, could induce cellular transformation with the standard assay of c-Ha-ras cotransfection into REFs (Schutte et al. 1989a,b) (Fig. 3). When cotransfected with activated ras,
Fos and Jun yielded refractile, transformed foci, with Fos being most active. In contrast, GCN4 /and all derivatives tested) gave essentially no transformed foci despite its ability to activate AP-1 site-dependent transcription in transient or stably transfected REFs. Thus, it is clear that cellular transformation cannot depend simply on the overall level of AP-1 transcriptional stimulation.

The amino-terminal regions of Jun and Fos stimulate transformation independently

To determine the functional differences between GCN4 and the Jun and Fos oncogenes, we generated Jun-GCN4 and Fos-GCN4 hybrid proteins by reciprocal exchange of their activation domains, basic regions, or leucine zippers (Fig. 4). For each derivative, transcription and transformation assays were carried out in the presence of $\mathrm{v}$-ras on equivalent populations of transfected cells. After normalizing for transfection efficiency, the relative transcription and transformation activities of each derivative were determined.

We first considered the possibility that the functional distinctions might reflect differences in DNA-binding activities especially because Fos/Jun and Jun/Jun complexes induce topological changes in the DNA (Kerppola and Curran 1991), whereas GCN4 does not (Gartenberg et al. 1990; Weiss et al. 1990). The substitution of the 
Table 1. Stable clones of Fos, Jun, and GCN4 activate AP-1 CAT constructs

\begin{tabular}{|c|c|c|}
\hline A. & Construct & $\begin{array}{l}\text { Number of independent } \\
\text { G418 colonies }\end{array}$ \\
\hline & Control & 140 \\
\hline & Fos & 220 \\
\hline & Jun & 180 \\
\hline & GCN4 & 190 \\
\hline & C/EBP & 0 \\
\hline B. & Stable pools & CAT conversion $(\%)$ \\
\hline & Control pool 1 & 5 \\
\hline & Control pool 2 & 6 \\
\hline & Control pool 3 & 3 \\
\hline & Control pool 4 & 9 \\
\hline & GCN4 pool 1 & 43 \\
\hline & GCN4 pool 2 & 75 \\
\hline & GCN4 pool 3 & 49 \\
\hline & GCN4 pool 4 & 67 \\
\hline & Fos pool 1 & 29 \\
\hline & Fos pool 2 & 77 \\
\hline & Fos pool 3 & 40 \\
\hline & Fos pool 4 & 83 \\
\hline & Jun pool 1 & 68 \\
\hline & Jun pool 2 & 45 \\
\hline & Jun pool 3 & 32 \\
\hline & Jun pool 4 & 54 \\
\hline
\end{tabular}

(A) G418-resistant colonies stably expressing the respective DNAs were generated as described in Materials and methods by use of a DNA/neo ratio of $15: 1$. Colonies were scored and either subcloned or pooled $14-21$ days post-transfection. $(B)$ G418-resistant colonies were isolated and transfected with the $5 \times$ TRE/tk-CAT construct. Transcriptional activation was assayed as described in Materials and methods.

GCN4 basic region into Jun and Fos (molecules $\mathrm{f} 1$ and $\mathrm{j} 2$; Fig. 4) should generate chimeric molecules with the binding specificity of GCN4 but with the activation and dimerization specificities of Jun or Fos. Examination of these hybrid proteins for transcriptional activity and oncogenic transformation (Figs. 4 and 5 ) indicates that the GCN4 basic region can functionally substitute for the basic regions of Jun and Fos in both assays. This indicates that the failure of GCN4 to transform cells is probably not related to subtle discrimination of AP-1 sequences in target genes.

To address the potential role of heterodimeric interactions, we analyzed chimeric molecules $\mathrm{f} 2$ and $\mathrm{j} 3$ in which the Fos and Jun leucine zipper are replaced by the GCN4 leucine zipper. These molecules, which should retain their own sequence specificity but be able to bind DNA only as homodimers, also induce cellular transformation. This observation indicates that interactions between Fos and Jun are not necessary for oncogenesis, and it suggests that Fos and Jun independently have transforming activity.

Although functional, molecules $\mathrm{fl}, \mathrm{f} 2, \mathrm{j} 2$, and $\mathrm{j} 3$ showed relatively low transcriptional and transforming activities (Figs. 4 and 5), presumably reflecting reduced
DNA-binding affinities of the hybrid bZIP domains. We therefore generated additional Jun or Fos chimeric molecules in which the intact bZIP domains were substituted as a single unit. The resulting chimeras containing either the Jun or Fos amino terminus and the GCN4 bZIP domain ( $j 4$ and $\mathrm{f} 3$ ) induced both transcriptional activation and cellular transformation to levels comparable or even better than the wild-type molecules (Figs. 4 and 5). Conversely, a chimera containing the GCN4 activation domain fused to the bZIP domain of Jun (j1) activates AP-1-dependent transcription but fails to induce cellular transformation. To further map the Fos region(s) involved in cellular transformation, we fused amino-terminal (f4) and carboxy-terminal (f5) regions to the GCN4 bZIP domain. f5 is more effective at activation of AP- 1 transcription than $\mathrm{f} 4$, although $\mathrm{f} 4$ is a more effective inducer of focus formation. The chimeric molecules $\mathrm{f} 3, \mathrm{f} 4$, and $\mathrm{j} 4$ also permit the formation of colonies in soft agar, and REFs stably transfected with these molecules cause aggressive tumor formation upon injection in athymic nude mice (data not shown).

To demonstrate that the GCN4 chimeras described above induced cellular transformation as homodimers, we substituted the GCN4 bZIP domain of molecule j4 with the bZIP domain derived from the Gl derivative, which has 7 extra amino acids between the basic region and the leucine zipper. Although the resulting molecule, $j 5$, has a different dimerization specificity from $j 4$, these derivatives induce transcriptional and transforming activities to comparable levels. These activities are inhibited by the introduction of an amino acid substitution (N235E) in the basic domain that inhibits DNA binding (i6). Taken together, these results demonstrate that the stimulation of AP-1-dependent transcription is not sufficient for cellular transformation. Both c-Jun and c-Fos carry information in their amino termini that do not stimulate generic AP-1 transcription better than the activation domain of GCN4, but these domains of Fos and (independently Jun) have the special property of inducing cellular transformation.

\section{Discussion}

Transcriptional activation through AP-1 sites is not sufficient for cellular transformation

The complex interactions that occur within and between different families of transcription factors are necessary for multicellular organisms to respond to the vast array of hormonal and developmental stimuli. A few specific domains are used by multiprotein families to promote the dimerization that is often necessary to bind to target DNA sequences with high affinity. In addition, interactions between different transcription factor families can permit "cross talk" between different regulatory circuits. However, this great complexity raises a significant challenge in the mechanistic interpretation of data obtained after introducing transcription factors into cells. It is extremely difficult to trace the multiple proteinprotein and protein-DNA interactions in which a single factor might participate. 

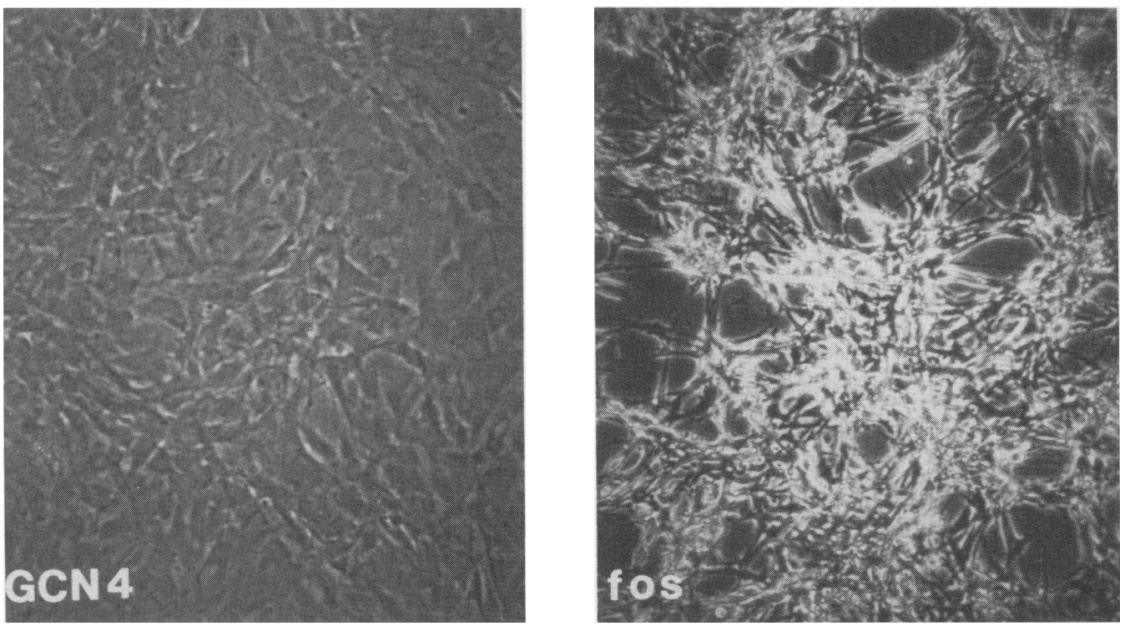

Figure 3. GCN4 does not transform REFs. REFs were transfected with $10 \mu \mathrm{g}$ of activated ras and each DNA construct, respectively. Stable foci were produced after 10-14 days in culture. The cells were fixed and stained with methylene blue. A representitive field of cells for each DNA construct is shown.

To circumvent these problems, we have expressed a heterologous and autonomous AP-1 transcription factor, yeast GCN4, in mammalian cells to address three critical issues relating to the function of the mammalian AP-1 protein family. First, is there an obligatory relationship between AP-1-dependent transcription and AP-1-dependent malignant transformation? Second, what is the inherent transforming potential of c-Jun and c-Fos under situations in which they cannot interact? Third, what is the nature of the oncogenically relevant regulatory regions of Fos and/or Jun?

Our results clearly demonstrate that increased levels of AP-1-dependent gene activation do not necessarily lead to cell transformation. When expressed in REFs, GCN4 stimulates transcription from artificial and natural promoters with an efficiency comparable to that of Jun and/or Fos. In striking contrast to Jun and Fos, expression of GCN4 does not yield transformed foci to a detectable extent, despite the facts that GCN4 does not appear to be cytostatic or cytotoxic and can chronically activate target genes in recovered G418-resistant colonies (Table 1). This leads to the conclusion that strong AP-1 site-dependent transcription does not necessarily lead to transformation.

\begin{tabular}{|c|c|c|c|}
\hline name & construct & $\begin{array}{l}\text { relative } \\
\text { transcriptional } \\
\text { activity }\end{array}$ & $\begin{array}{l}\text { relative } \\
\text { transforming } \\
\text { activity }\end{array}$ \\
\hline GCN4 & activation basic dimenzation & 100 & $<2$ \\
\hline jun & 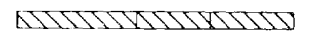 & 53 & 50 \\
\hline fos & 1 & 65 & 100 \\
\hline$f 1$ & $\square$ & 38 & 50 \\
\hline 12 & & 16 & 20 \\
\hline$\uparrow 3$ & $\square$ & 45 & 70 \\
\hline 14 & N.tos & 22 & 80 \\
\hline$\uparrow 5$ & $c \cdot \cos$ & 40 & 25 \\
\hline j1 & 1110118 & 37 & 4 \\
\hline j2 & $8111111-91118$ & 26 & 15 \\
\hline$\beta$ & 5717.181111 & 27 & 20 \\
\hline$j 4$ & 8.11112 & 75 & 70 \\
\hline$j 5$ & 811118 & 66 & 60 \\
\hline$j 6$ & $85111111-a$ & 14 & $<3$ \\
\hline control & & 12 & $<2$ \\
\hline
\end{tabular}

Figure 4. Amino-terminal regions of Fos and Jun are necessary for cellular transformation. A schematic diagram of the different Fos/GCN4 and Jun/GCN4 chimeric molecules is shown. Each chimeric construct was tested in the presence of c-Ha-ras for both AP-1dependent transcriptional activity $(5 \times \mathrm{TRE} / \mathrm{tk}-\mathrm{CAT})$ and foci formation. Each construct was prepared in duplicate and double banded on a cesium chloride gradient. These numbers represent at least three to six independent transfection. 

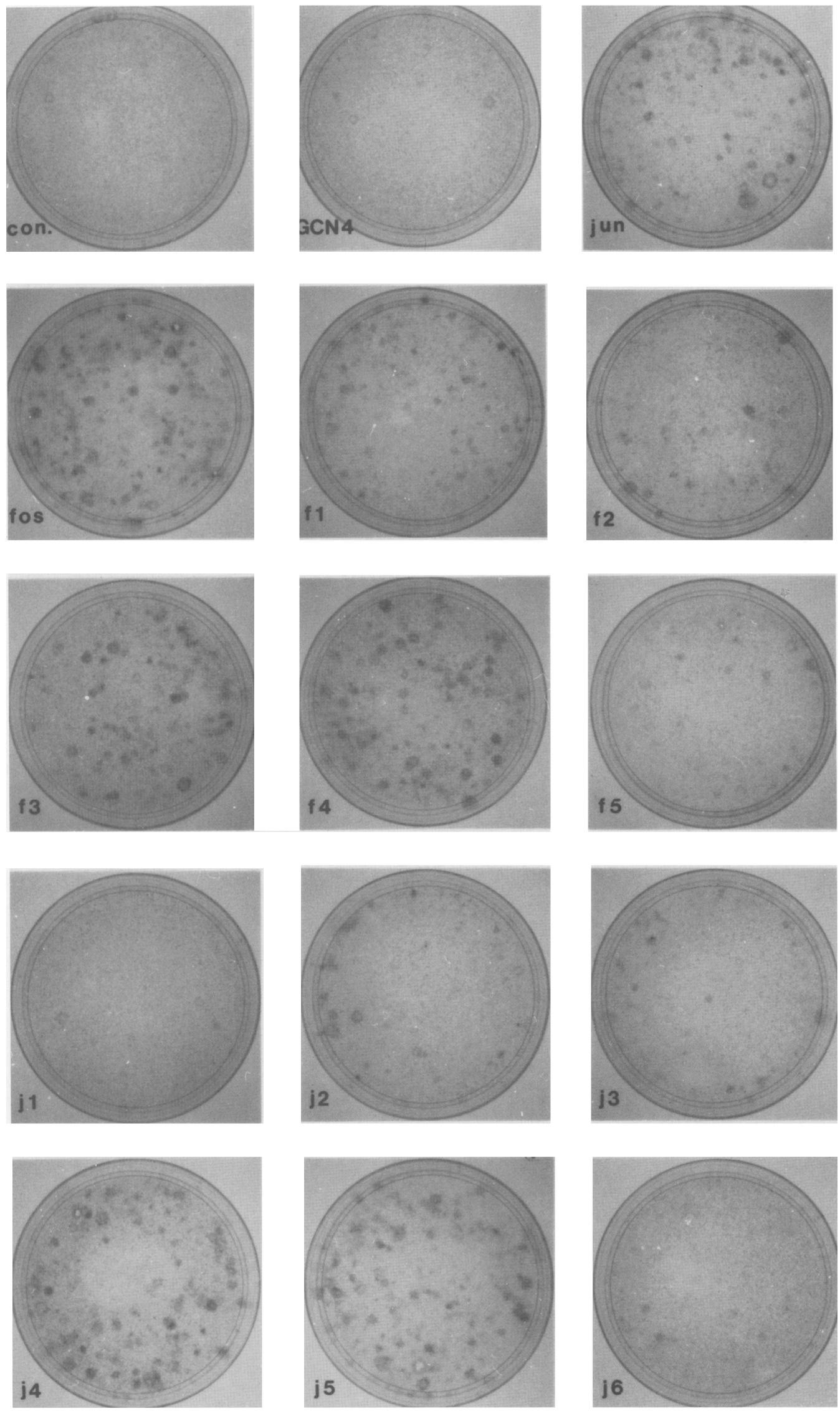

Figure 5. Homodimerization of Fos/GCN4 and Jun/GCN4 chimeric molecules can induce stable transformation. REFs were transfected with each chimeric construct $(10 \mu \mathrm{g})$ and activated ras $(10 \mu \mathrm{g})$. Stable transformants were visible after 10-14 days. Cells were fixed and stained with methylene blue. A representative field of cells transfected with each chimeric construct is shown. 
ras plays an important role in AP-1-dependent transformation, and it also affects the phosphorylation of the amino-terminal region of Jun, which increases its transcriptional activity (Binetruy et al. 1991). However, our assays of transcriptional activation and cellular transformation are carried out in the presence of ras on identical populations of transfected cells. Thus, the role of ras in cellular transformation cannot be explained simply as increased AP-1-dependent transcriptional activity. Instead, it seems likely that ras-dependent phosphorylation might be necessary for the oncogenic regulatory function of Jun (and perhaps Fos).

\section{The amino-terminal regions of Fos and Iun have inherent transformation potential}

AP-1 family members interact with each other and with other transcription factor families, making it difficult to know whether Jun or Fos individually has transforming activity or whether the heterotypic interactions of each are necessary for transformation. Because the GCN4 bZIP domain strongly favors homodimer formation, chimeras between this domain and other portions of Jun or Fos may reasonably be assumed to result in homodimers in vivo. Furthermore, transcription and transformation activities are not affected by the insertion of 7 residues between the leucine zipper and the basic region of GCN4, which retains wild-type DNA-binding affinity as a homodimer but alters potential heterodimeric partners (Pu and Struhl 1991b). Thus, from the observation that Jun-GCN4 and Fos-GCN4 homodimers transform cells efficiently, we conclude that c-Jun and c-Fos each contain inherent transforming activity.

As the various members of the AP-1 transcription factor family might subtly discriminate between genomic AP-1 sites, it is possible that GCN4 fails to induce cellular transformation because it binds poorly to that subset of genes involved in cell proliferation that are capable of being activated by AP-1. However, this hypothesis is excluded by the properties of the Fos and Jun chimeras containing the GCN4 bZIP domain. In these cases $(\mathrm{f} 3$, $\mathrm{f} 4$, j4), all of the information needed to recognize AP-1 sites is provided by GCN4, yet cellular transformation is induced with an efficiency comparable to that of Jun or Fos. Transformation by these GCN4 chimeras requires AP-1 DNA-binding activity because foci are not observed upon transfection of a derivative containing a mutated bZIP domain (j6).

\section{Mechanistic implications}

Our results clearly indicate that the amino-terminal regions of Jun and Fos each contain special regulatory functions that are required for cellular transformation but are mechanistically distinct from generic transcriptional activation domains. Although these Fos and Jun regions are no better than the GCN4 activation domain at stimulating transcription from the promoters tested, it is conceivable that the oncogenically relevant AP- 1 site containing a target gene has special requirements for tran- scriptional activation by the AP-1 family. For example, activation of the key transforming gene might require a protein-protein interaction between Fos or Jun and some other specific transcription factor that binds /directly or indirectly) to the promoters. Presumably, such interactions would involve the amino termini of Fos or Jun and would not occur with the GCN4 activation domain. Another possibility is that Fos and Jun might differ from GCN4 in interacting with the basal transcription machinery at specific promoters; in this regard, certain TATA elements respond differentially to upstream activator proteins (Struhl 1986; Homa et al. 1988; Simon et al. 1988; Harbury and Struhl 1989; Wefald et al. 1990). All of the above models are consistent with the mutational analyses of Jun and Fos in which transcriptional activity correlates well with transformation ability (Bohmann and Tjian 1989; Neuberg et al. 1989b; Schuermann et al. 1989; Baichwal and Tiian 1990; Alani et al. 1991; Binetruy et al. 1991; Lucibello et al. 1991; Wisdom et al. 1992). However, although AP-1 transcriptional activity may be important or even necessary for transformation, it is not sufficient.

Alternatively, transformation by Fos and Jun might require AP-1-dependent repression of one or more tumor suppressor genes, especially as Fos and Jun can each act negatively at certain promoters containing AP- 1 sites. In this view, GCN4 would function well as a positive activator but be incapable of acting negatively. A very recent study of Jun derivatives with mutations in the aminoterminal $\delta$ region shows an inverse correlation between transforming ability and transcriptional activity, leading to the suggestion of a negative role for Jun in transformation (Havarstein et al. 1992). Although our data show no correlation between the relative transcriptional activities of the various derivatives and their transforming activities, the possibility of transformation by a negative function of Jun and Fos is not excluded.

Our results are also useful in addressing which of the various functions of Jun and Fos might be relevant to cellular transformation. For example, the bZIP domains of Jun and Fos can interact directly with the glucocorticoid receptor (Schule et al. 1990; Yang-Yen et al. 1990) and with myoD (Bengal et al. 1992), generally (though not always/ resulting in transcriptional repression. However, such interactions seem unlikely to be relevant for oncogenesis because the heterologous GCN4 and G1 bZIP domains are very compatible with cellular transformation. On the other hand, the amino terminus of Jun can mediate repression of transcriptional activation by the estrogen receptor (Doucas et al. 1991) and by myogenin or MyoD (Li et al. 1992); this suggests the possibility that transformation involves similar mechanisms to those utilized in suppressing the function of transcription factors that are necessary for establishing or maintaining the differentiated states of the cell.

Finally, it should be noted that the various constructs used here may be helpful in identifying AP-1-regulated genes that are involved in cellular transformation. Whatever mechanisms distinguish wild-type GCN4 from Fos or Jun, there must be differences in their abilities to 
activate or repress certain crucial genes. By cloning mRNAs differentially regulated by GCN4 versus a GCN4 derivative with transforming activity, it might be possible to identify target genes that are centrally involved in AP-1-dependent malignant transformation.

\section{Materials and methods}

\section{DNA constructs}

Human c-Jun cDNA, rat c-Fos cDNA, and activated cHa-ras (pEJ6.6) were obtained respectively, from R. Tjian (University of California, Berkeley), T. Curran (Roche Institute of Molecular Biology, Nutley, NI), and R. Weinberg (MIT, Cambridge, MA). All constructs were cloned into the BamHI site of the mammalian expression vector pMTpn, which contains the SV40 early/ late enhancer, the human metallothionine HIIa promoter, and the human growth hormone 3 '-untranslated region /Greene et al. 1986). 5× TRE/tk-CAT (obtained from M. Karin, University of California, San Diego/ contains five copies of the collagenase AP-1 sequence linked to the tk-CAT. The GCN4-coding sequence contained a BamHI restriction site $5^{\prime}$ of the basic domain and a $X$ hoI restriction site at the first leucine of the dimerization domain (Pu and Struhl 1991). To permit efficient translational initiation in mammalian cells, we modified the region surrounding the GCN4 translational initiation codon by cloning TGCACGCCACCATGGTACCAAGTTTATTTGCT at the Dral site; the native GCN4 gene was poorly translated in mammalian cells. The GCN4 mutant G1 was obtained by subcloning the BamHI-SacI fragment, which contains the 7 amino-acid insertion at amino acid 252. G2 was obtained by an in-frame deletion of the BamHI restriction fragment containing the entire activation domain. G3 was obtained by restricting pMTpnGCN4 with $X b a I$ and $X h o I$, blunting the ends, and religating. G4 was obtained by deleting the DNA between the XhoI site and the SacI site, leaving the TGA stop codon in-frame. G5 was generated by subcloning the BamHI-SacI restriction fragment derived from the GCN4-Glu-235 derivative (Pu and Struhl 1991 ! into the GCN4-pMTpn.

\section{Chimeric constructs}

The different chimeric Jun-GCN4 and Fos-GCN4 constructs were generated by inserting in-frame restriction sites without altering the amino acids sequence. DNA fragments were amplified by use of the polymerase chain reaction (PCR) and subcloned into the specific regions of each gene. All chimeric molecules were confirmed by DNA sequencing.

Jun chimeras jl was obtained by amplifying the region containing the Jun bZIP domain with oligonucleotides A (CCCTCTAGAGCCTCAGACAGTGCCGCAG) and B (CCCGAGCTCAAAATGTTTGCAACTGCTGCGTTAGCATG), cleaving the resulting product with $\mathrm{XbaI}$ and $\mathrm{SacI}$, and ligating the fragment into the GCN4 pMTpn plasmid. j4 was obtained by amplifying the Jun amino-terminal domain with oligonucleotides C (CCCGTCGACCATGGAAACGACCTTCTATGACGATGCC) and D (CCCGGATCCCTCCTGGGACTCCATGTC), cleaving the resulting product with SalI and BamHI, and inserting the fragment into pMTpn-GCN4. The $i 2$ molecule was obtained by amplification of the Jun dimerization region with oligonucleotide E (CCCCTCGAGGAAAAAGTGAAAACCTTGAAAGC) and $B$, and the fragment was subcloned into the chimeric molecule $\mathrm{j} 4$. The $\mathrm{j} 3$ molecule was obtained by subcloning the amplified Jun dimerization domain into the pMTpn-GCN4 molecule. The i5 molecule was obtained by sub- cloning the BamHI-SacI fragment from the mutant G2 into the same sites of the chimeric $\mathrm{j} 3$ molecule. $\mathrm{j} 6$ was cloned as $\mathrm{j} 5$; however, the BamHI-SacI fragment was obtained from the mutant G3.

Fos chimeras The $\mathrm{fl}$ chimera was obtained by substitution of the GCN4 basic region with the Fos basic domain in two successive subcloning reactions. The Fos carboxy-terminal domain was amplified with $F$ (CCCAAGCTTCTCGAGGCGGAGACAGATCAACTTGAAGAC) and G (CCAAGGCCAGGAGAGGCACT); the resulting product was cleaved with HindIII and $\mathrm{SacI}$ and subcloned into the vector pBluescript II KS to generate intermediate molecule SO68. The GCN4 basic domain was amplified with oligonucleotide $\mathrm{H}$ (CCCGTCGACGGATCCTCGTGCTCTAAAACGTGCTGC), and the resulting SalI-BamHI fragment was cloned into SO68. Finally, the SalI-SacI fragment containing the GCN4 basic and Fos dimerization domain was subcloned into the pMTpn-Fos vector. The $\mathrm{f} 2$ chimera was obtained by amplifying the amino terminus of the Fos molecule with oligonucleotides I (AGAGCGCCCCATCCTTACGG) and I (GCCTCGAGCGTATCTGTCAGCTCCCT); and following digestion with SalI and $\mathrm{XhoI}$, the fragment was subcloned into the pGEM vector (SO75). The carboxyl terminus of Fos in this reaction was obtained with oligonucleotides $M$ /CCCCAAGC TTGTGGGTGAGCGTGAAAAACTGGAGTTTATTTTGGC$A G C C)$ and $G$, and the resulting DNA fragment was subcloned into an intermediate pMTpn-GCN4 vector (SO74). The complete fragment containing the GCN4 dimerization domain fused to the Fos carboxyl terminus was subcloned into the SalISacI restriction site of the pMTpn-Fos vector. The $\mathrm{f} 3$ chimera was obtained by subcloning the XhoI-SacI fragment derived from the $\mathrm{f} 2$ chimera into the same sites of the $\mathrm{fl}$ chimera. The f4 molecule was obtained by subcloning the GCN4 dimerization domain into the vector (SO68). The GCN4 basic and dimerization regions were then subcloned into the pMTpn-Fos molecule as a SalI-SacI fragment. The 5 molecule was obtained by cloning the amplified Fos carboxyl terminus used to create the f2 chimera downstream of a synthetic traditional start site containing an in-frame ATG, the amino acids for the influenza epitope, and a HindIII site in the same reading frame as the Fos carboxyl terminus (SO73). The SacI site of the Fos carboxyterminal and the BamHI site in the GCN4 molecule were blunt ended, and the GCN4 basic and zipper domains were then subcloned in-frame 3 ' of the Fos molecule.

\section{Cell culture}

REFs were isolated as described previously (Schutte et al. 1989a). Briefly, 14- to 15-day Fisher rat embryos were sacrificed, rinsed, and trypsinized for $30 \mathrm{~min}$ at $37^{\circ} \mathrm{C}$. Culture medium [Dulbecco's modified Eagle (DME) supplemented with glutamine, pennicillin-streptomycin, and $10 \%$ fetal calf serum] was added, and the cells were dispersed, counted, and plated on $100-\mathrm{mm}$ tissue culture dishes (Costar) at a density of $2 \times 10^{6}$ to $4 \times 10^{6} /$ dish. The cells were refed with culture medium after 12 $\mathrm{hr}$, and trypsinized after $24-36 \mathrm{hr}$, and aliquots were frozen in liquid nitrogen. For experiments, REFs were plated at $1 \times 10^{5}$ to $2 \times 10^{5} / 100$-mm tissue culture dish and allowed to reach $80-$ $90 \%$ confluence ( $1-2$ days). All DNAs used in cell transfections were doubly banded on cesium chloride gradients followed by ethanol precipitation, and at least two different DNA preparations of each construct were independently transfected.

\section{CAT assays}

Confluent REFs $(80-90 \%)$ were fed with culture medium 1-2 hr before transfection. For the experiment in Figure 2, $10 \mu \mathrm{g}$ of 
each construct was cotransfected with $2 \mu \mathrm{g}$ of $5 \times \mathrm{TRE} / \mathrm{tk}-\mathrm{CAT}$ and $1.5 \mu \mathrm{g}$ of $\beta$-actin-lacZ with standard calcium phosphate precipitation techniques. After 6-8 hr the cells were washed with PBS and refed with culture medium. The cells were harvested $40-48 \mathrm{hr}$ after transfection, and the extract was prepared as described by Gorman et al. (1982). $\beta$-Galatosidase activity was determined for each extract, and all CAT assays were normalized for equivalent transfection efficiency. For the experiment in Figure 4, the same procedure was followed, except that $10 \mu \mathrm{g}$ of activated Ha-ras was also cotransfected (see below).

\section{Foci formation}

Confluent REFs $(80-90 \%$ ) were fed with culture medium $1-2 \mathrm{hr}$ before to transfection. Ten micrograms of each construct was cotransfected with $10 \mu \mathrm{g}$ of activated Ha-ras, $2 \mu \mathrm{g}$ of $5 \times$ TRE/ tk-CAT, and $1.5 \mu \mathrm{g}$ of $\beta$-actin-lac $Z$ with the standard calcium phosphate techniques. The cells were rinsed with PBS and refed with culture medium $6-8 \mathrm{hr}$ post-transfection. The cells were trypsinized $48 \mathrm{hr}$ after transfection and split $1: 3$. One of the plates was used to measure both the $\beta$-galactosidase activity and the levels of AP-1 transcriptional activation, as measured by CAT conversion. The remaining cells were left to reach confluence, at which time they were refed with culture medium containing $5 \%$ fetal calf serum and refed every 2 days thereafter. Stable cell transformants (foci) were visible after 7-10 days, and the numbers of foci were normalized for transfection efficiency by $\beta$-galactosidase activity. Foci generated were subjected to soft agar growth test. A pool of foci (plate) for several constructs $(\mathrm{j} 4, \mathrm{f} 3, \mathrm{f} 4)$ were injected into nude mice, and tumors were observed from 8 to 25 days later.

\section{Acknowledgments}

We thank William Pu and Chris Brandl for the GCN4 mutant DNAs, Robert Distel for the aP2 and aP2 mutant clones, and Charles Stiles and Rolf Freter for their help in quantitating CAT activities with the PhosphorImager. S.O. was supported by a postdoctoral fellowship from the Damon Ramon-Walter Winchell Foundation and G.S.R. was supported by a postdoctoral fellowship from the National Research Council at the National Institutes of Health (NIH). The work was supported by NIH grants to B.M.S. (HD 24926) and K.S. (GM30186, GM46555), by Grant Associazione Italiana per Ricerca sul Cancro (A.I.R.C.), and by the Lucille Markey trust.

The publication costs of this article were defrayed in part by payment of page charges. This article must therefore be hereby marked "advertisement" in accordance with 18 USC section 1734 solely to indicate this fact.

\section{References}

Agre, P., P.F. Johnson, and S.L. McKnight. 1989. Cognate DNA binding specificity retained after leucine zipper exchange between GCN4 and C/EBP. Science 246: 922-926.

Alani, R., P. Brown, B. Binetruy, H. Dosaka, R.K. Rosenberg, P. Angel, M. Karin, and M.J. Birrer. 1991. The transactivating domain of the c-Jun proto-oncoprotein is required for cotransformation of rat embryo cells. Mol. Cell. Biol. 11: 6286-6295.

Angel, P., M. Imagawa, R. Chiu, B. Stein, R.J. Imbra, H.J. Rahmsdorf, C. Jonat, P. Herrlich, and M. Karin. 1987. Phorbol ester-inducible genes contain a common cis element recognized by a TPA-modulated trans-acting factor. Cell 49: 729-739.
Angel, P., E.A. Allegretto, S.T. Okino, K. Hattori, W.J. Boyle, T. Hunter, and M. Karin. 1988. Oncogene jun encodes a sequence-specific trans-activator similar to AP-1. Nature 332: $166-171$.

Angel, P., K. Hattori, T. Smeal, and M. Karin. 1988. The iun proto-oncogene is positively autoregulated by its product. Cell 55: 875-885.

Baichwal, V.R. and R. Tiian. 1990. Control of c-Jun activity by interaction of a cell-specific inhibitor with regulatory domain $\delta$ : Differences between v- and c-Jun. Cell 63: 815-825.

Bengal, E., L. Ransone, R. Scharfmann, V.J. Dwarki, S.J. Tapscott, H. Weintraub, and I.M. Verma. 1992. Functional antagonism between c-Jun and MyoD proteins: A direct physical association. Cell 68: 507-519.

Binetruy, B., T. Smeal, and M. Karin. 1991. Ha-ras augments c-jun activity and stimulates phosphorylation of its activation domain. Nature 351: 122-127.

Bohmann, D. and R. Tiian. 1989. Biochemical analysis of transcriptional activation by Jun: Differential activity of c- and v-Jun. Cell 59: 709-717.

Bohmann, D., T.J. Bos, A. Admon, T. Nishimura, P.K. Vogt, and R. Tjian. 1987. Human proto-oncogene c-iun encodes a DNA binding protein with structural and functional properties of transcription factor AP-1. Science 238: 1386-1392.

Boyle, W.J., T. Smeal, L.H.K. Defize, P. Angel, J.R. Woodgett, M. Karin, and T. Hunter. 1991. Activation of protein kinase C decreases phosphorylation of c-Jun at sites that negatively regulate its DNA-binding activity. Cell 64: 573-584.

Brenner, D.A., M. O'Hara, P. Angel, M. Chojkier, and M. Karin. 1989. Prolonged activation of jun and collagenase genes by tumour necrosis factor- $\alpha$. Nature 337: 661-663.

Castellazzi, M., G. Spyrou, N. La Vista, J.-P. Dangy, F. Piu, M. Yaniv, and G. Brun. 1991. Overexpression of c-jun, junB, or junD affects cell growth differently. Proc. Natl. Acad. Sci. 88: 8890-8894.

Chiu, R., P. Angel, and M. Karin. 1989. Jun-B differs in its biological properties from, and is a negative regulator of, $c$-Jun. Cell 59: 979-986.

Curran, T. and B.J. Franza. 1988. Fos and Jun: The AP-1 connection. Cell 55: 395-397.

Curran, T., G. Peters, C. VanBeveren, N. Teich, and I. Verma. 1982. FBJ murine osteosarcoma virus: Identification and molecular cloning of biologically active proviral DNA. J. Virol. 44: 674-682.

Diamond, M., J.N. Miller, S.K. Yoshinaga, and K.R. Yamamoto. 1990. Transcription factor interactions: Selectors of positive or negative regulation from a single DNA element. Science 249: 1266-1272.

Distel, R., H.S. Ro, B.S. Rosen, D. Groves, and B.M. Spiegelman. 1987. Nucleoprotein complexes that regulated gene expression in adipocyte differentiation: Direct participation of c-fos. Cell 49: 835-844.

Doucas, V., G. Spyrou, and M. Yaniv. 1991. Unregulated expression of c-Jun or c-Fos but not JunD inhibits estrogen receptor activity in human breast cancer derived cells. EMBO $\mathrm{J}$. 10: $2237-2245$.

Gartenberg, M.R., C. Ampe, T.A. Steitz, and D.M. Crothers. 1990. Molecular characterization of the GCN4-DNA complex. Proc. Natl. Acad. Sci. 87: 6034-6038.

Gorman, C.M., L.F. Moffat, and B.H. Howard. 1982. Recombinant genomes which express chloramphenicol acetyltransferase in mammalian cells. Mol. Cell. Biol. 2: 1044-1051.

Greene, G.L.P., P. Gilna, M. Waterfield, A. Baker, Y. Hort, and J. Shine. 1986. Sequence and expression of human estrogen receptor complementary DNA. Science 231: 1150-1154.

Halazonetis, T.D., K. Georgopoulos, M.E. Greenberg, and P. Le- 
der. 1988. c-Jun dimerizes with itself and with c-Fos, forming complexes of different DNA binding affinities. Cell 55: 917924.

Harbury, P.A.B. and K. Struhl. 1989. Functional distinctions between yeast TATA elements. Mol. Cell. Biol. 9: 52985304.

Havarstein, L.S., I.M. Morgan, W.-Y. Wong, and P.K. Vogt. 1992. Mutations in the Jun Delta region suggest an inverse correlation between transformation and transcriptional activation. Proc. Natl. Acad. Sci. 89: 618-622.

Herrera, R., H.S. Ro, G.S. Robinson, K.G. Xanthopoulos, and B.M. Spiegelman. 1989. A direct role for C/EBP and the AP-1 binding site in gene expression linked to adipocyte differentiation. Mol. Cell. Biol. 9: 5331-5339.

Hill, D.E., I.A. Hope, J.P. Macke, and K. Struhl. 1986. Saturation mutagenesis of the yeast HIS3 regulatory site: requirements for transcriptional induction and for binding by GCN4 activator protein. Science 234: 451-457.

Homa, F.L., J.C. Glorioso, and M. Levine. 1988. A specific 15-bp TATA box promoter element is required for expression of a herpes simplex virus type I late gene. Genes \& Dev. 2: 4053.

Jonat, C., H.J. Rahmsdorf, K.-K. Park, A.C.B. Cato, S. Gebel, H. Ponta, and P. Herrlich. 1990. Antitumor promotion and antiinflammation: Down-modulation of AP-1 (Fos-Jun) activity by glucocorticoid hormone. Cell 62: 1189-1204.

Kerppola, T.K. and T. Curran. 1991. Fos-Jun heterodimers and Jun homodimers bend DNA in opposite orientations: Implications for transcription factor cooperativity. Cell 66: 317326.

Konig, H., H. Ponta, U. Rahmsdorf, M. Buscher, A. Schonthal, H.J. Rahmsdorf, and P. Herrlich. 1989. Autoregulation of fos: The dyad symmetry element as the major target of repression. EMBO J. 8: 2559-2566.

Kouzarides, T. and E. Ziff. 1988. The role of the leucine zipper in the fos-jun interaction. Nature 336: 646-651.

- 1989. Leucine zippers of fos, jun, and GCN4 dictate dimerization specificity and thereby control DNA binding. Nature 340: $568-571$.

Kozak, M. 1987. An analysis of 5 '-noncoding sequences from 699 vertebrate messenger RNAs. Nucleic Acids Res. 15: $8125-8148$.

Landschulz, W.H., P.F. Johnson, and S.L. McKnight. 1988. The leucine zipper: A hypothetical structure common to a new class of DNA binding proteins. Science 240: 1759-1764.

Lee, W., P. Mitchell, and R. Tiian. 1987. Purified transcription factor AP-1 interacts with TPA-inducible enhancer elements. Cell 49: 741-752.

Li, L., J.S. Hu, and E.N. Olson. 1990. Different members of the jun family exhibit distinct patterns of expression in response to type $\beta$ transforming growth factor. 1 . Biol. Chem. 265: 1556-1562.

Li, L., J.-L. Chambard, M. Karin, and E.N. Olson. 1992. Fos and Jun repress transcriptional activation by myogenin and MyoD: The amino terminus of Jun can mediate repression. Genes \& Dev. 6: 676-689.

Lucibello, F.C., M. Neuberg, T. Jenuwein, and R. Muller. 1991. Multiple regions of $\mathrm{v}$-fos protein involved in the activation of AP-1 dependent transcription: Is trans-activation crucial for transformation. New Biol. 3: 671-677.

Maki, Y., T.J. Bos, C. Davis, M. Starbuck, and P.K. Vogt. 1987. Avian sarcoma virus 17 carries a new onc gene, iun. Proc. Natl. Acad. Sci. 84: 2848-2852.

Miller, A.D., T. Curran, and I.M. Verma. 1984. c-fos protein can induce cellular transformation: A novel mechanism of activation of a cellular oncogene. Cell 36: 51-60.
Mumberg, D., F.C. Lucibello, M. Schuermann, and R. Muller. 1991. Alternative splicing of $f O S B$ transcripts results in differentially expressed mRNAs encoding functionally antagonistic proteins. Genes \& Dev. 5: 1212-1223.

Nakabeppu, Y. and D. Nathans. 1991. A naturally occuring truncated form of FosB inhibits Fos/Jun transcriptional activity. Cell 64: 751-759.

Nakabeppu, Y., K. Ryder, and D. Nathans. 1988. DNA binding activities of three murine Jun proteins: Stimulation by Fos. Cell 55: 907-915.

Neuberg, M., J. Adamkiewicz, J.B. Hunter, and R. Muller. 1989a. A Fos protein containing the Jun leucine zipper forms a homodimer which binds to the AP1 binding site. Nature 341: 243-245.

Neuberg, M., M. Schuermann, J.B. Hunter, and R. Muller. 1989b. Two functionally different regions in Fos are required for the sequence-specific DNA interaction of the Fos/Jun protein complex. Nature 338: 589-590.

Oliviero, S. and K. Struhl. 1991. Synergistic transcriptional enhancement does not depend on the number of acidic activation domains bound to the promoter. Proc. Natl. Acad. Sci. 88: $224-228$.

Pu, W.T. and K. Struhl. 1991a. Highly conserved residues in the bZIP domain of yeast GCN4 are not essential for DNA-binding. Mol. Cell. Biol. 11: 4918-4926.

- 1991b. The leucine zipper symmetrically positions the adjacent basic regions for specific binding to DNA. Proc. Natl. Acad. Sci. 88: 6901-6905.

Ransone, L.J. and I.M. Verma. 1990. Nuclear protooncogenes Fos and Jun. Annu. Rev. Cell Biol. 6: 539-557.

Schuermann, M., M. Neuberg, J.B. Hunter, T. Jenuwein, R.-P. Ryseck, R. Bravo, and R. Muller. 1989. The leucine repeat motif in Fos protein mediates complex formation with Jun/ AP-1 and is required for transformation. Cell 56: 507-516.

Schule, R., P. Rangarajan, S. Kliewer, L.J. Ransone, J. Bolado, N. Yang, I.M. Verma, and R.M. Evans. 1990. Functional antagonism between oncoprotein c-Jun and the glucocorticoid receptor. Cell 62: 1217-1226.

Schutte, J., J. Minna, and M. Birrer. 1989a. Deregulated expression of human c-iun transforms primary rat embryo cells in cooperation with an activated c-Ha-ras gene and transforms rat-l A cells as a single gene. Proc. Natl. Acad. Sci.. 86: $2257-$ 2261.

Schutte, J., J. Viallet, M. Nau, S. Segal, J. Fedorko, and J. Minna. 1989b. Jun-B inhibits and c-Fos stimulates the transforming and trans-activating activities of c-Jun. Cell 59: 987-997.

Sellers, J.W. and K. Struhl. 1989. Changing fos oncoprotein to a DNA-binding protein with GCN4 dimerization specificity by swapping "leucine zippers." Nature 341: 74-76.

Simon, M.C., T.M. Fisch, B.J. Benecke, J.R. Nevins, and N. Heintz. 1988. Definition of multiple, functionally distinct TATA elements, one of which is a target in the hsp 70 promoter for ElA regulation. Cell 52: 723-729.

Stein, B., H.J. Rahmsdorf, A. Steffen, M. Litfin, and P. Herrlich. 1989. UV induced DNA damage is an intermediate in the UV induced expression of HIV-1, collagenase, c-fos, and metallothionein. Mol. Cell. Biol. 9: 5169-5181.

Struhl, K. 1986. Constitutive and inducible Saccharomyces cerevisiae promoters: Evidence for two distinct molecular mechanisms. Mol. Cell. Biol. 6: 3847-3853.

1987. The DNA-binding domains of the jun oncoprotein and the yeast GCN4 transcriptional activator are functionally homologous. Cell 50: $841-846$.

- 1988. The JUN oncoprotein, a vertebrate transcription factor, activates transcription in yeast. Nature 332: 649-650. Umek, R.M., A.D. Friedman, and S.L. McKnight. 1991. 
CCAAT/enhancer binding protein: a component of a differentiation switch. Science 251: 288-292.

Wefald, F.C., B.H. Devlin, and R.S. Williams. 1990. Functional heterogeneity of mammalian TATA-box sequences revealed by interaction with a cell-specific enhancer. Nature 344: 260-262.

Weiss, M.A., T. Ellenberger, C.R. Wobbe, J.P. Lee, S.C. Harrison, and K. Struhl. 1990. Folding transition in the DNA-binding domain of GCN4 on specific binding to DNA. Nature 347: 575-578.

Wisdom, R., J. Yen, D. Rashid, and I.M. Verma. 1992. Transformation by FosB requires a trans-activation domain missing in FosB2 that can be substituted by heterologous activation domains. Genes \& Dev. 6: 667-675.

Yang-Yen, H.-F., J.-C. Chambard, Y.-L. Sun, T. Smeal, T. J. Schmidt, J. Drouin, and M. Karin. 1990. Transcriptional interference between c-Jun and the glucocorticoid receptor: Mutual inhibition of DNA binding due to direct proteinprotein interaction. Cell 62: 1205-1215.[uftx8] 


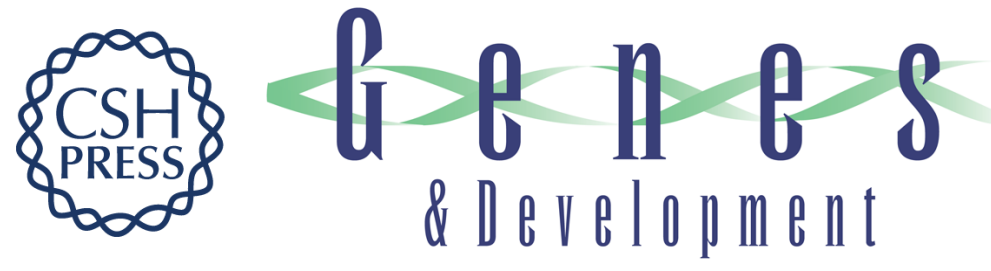

\section{Yeast GCN4 as a probe for oncogenesis by AP-1 transcription factors: transcriptional activation through AP-1 sites is not sufficient for cellular transformation.}

S Oliviero, G S Robinson, K Struhl, et al.

Genes Dev. 1992, 6:

Access the most recent version at doi:10.1101/gad.6.9.1799

References This article cites 65 articles, 27 of which can be accessed free at: http://genesdev.cshlp.org/content/6/9/1799.full.html\#ref-list-1

License

Email Alerting Service

Receive free email alerts when new articles cite this article - sign up in the box at the top right corner of the article or click here.

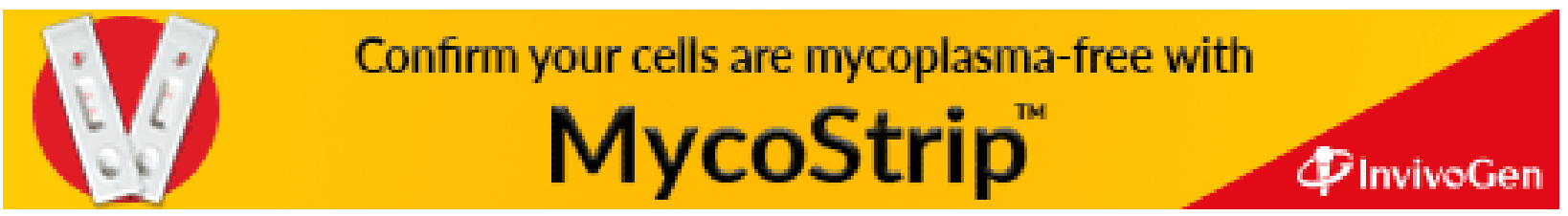

